DOI: 10.12957/demetra.2017.22391

\title{
Análise de flavonoides totais presentes em algumas frutas e hortaliças convencionais e orgânicas mais consumidas na região Sul do Brasil
}

\section{Analysis of total flavonoids present in some of the most consumed conventional and organic fruits and vegetables in southern Brazil}

\author{
Patrícia do Rocio Smolinski Savi ${ }^{1}$ \\ Larissa dos Santos' \\ Amanda Macedo Gonçalves' \\ Simone Biesek! \\ Cristina Peitz de Lima' \\ ' Centro Universitário Autônomo do Brasil, Escola \\ de Saúde, Curso de Nutrição. Curitiba-PR, Brasil. \\ Correspondência / Correspondence \\ Cristina Peitz de Lima \\ E-mail: cristinalima@unibrasil.com.br
}

\section{Resumo}

Os flavonoides existentes na natureza estão relacionados com grande variedade de atividades biológicas, como ação antiinflamatória e antioxidante, apresentando efeitos benéficos à saúde. O objetivo deste estudo foi analisar e comparar o teor de flavonoides totais presentes em frutas e hortaliças mais consumidas na região Sul do Brasil, segundo dados da Pesquisa de Orçamento Familiar POF - 2008/2009. Foram analisados cebola, tomate, banana e maçã, de cultivo orgânico e convencional, in natura, e submetidos ao processo de cocção em calor seco na panela e micro-ondas. A determinação de flavonoides totais foi realizada por método espectrofotométrico, utilizando cloreto de alumínio. Os resultados das análises de determinação de flavonoides totais correspondem à média \pm desvio padrão de três repetições. A maçã e a cebola apresentaram maior teor de flavonoides. $\mathrm{O}$ processo de cocção afetou a extração dos flavonoides nas frutas, diminuindo os teores, fato explicado pela presença das fibras. Já para as hortaliças, o processo facilitou a extração dos flavonoides. A recomendação diária de flavonoides não é definida, porém o profissional nutricionista, conhecendo como os alimentos devem ser consumidos e seus teores de flavonoides, pode prescrever e traçar estratégias e metas seguras de ingestão, para promoção da saúde do cliente, visando também a sua condição social e econômica.

Palavras-chave: Flavonoides. Frutas. Verduras. Alimentos Orgânicos. Produção Agrícola. Manipulação de Alimentos. 


\section{Abstract}

The flavonoids present in nature are related to a large variety of biological activities, such as anti-inflammatory and antioxidant action, and they have beneficial effects on health. The objective of this study was to analyze and compare the amount of flavonoids presented in the fruits and vegetable most consumed in southern Brazil, according to data of (family budget request survey) POF 2008/2009. Fresh onions, tomatoes, bananas and apples, both from conventional and organic cultivation, ere analyzed and submitted to dry heat cooking methods. Total flavonoid content was determined by the spectrophotometric method, using aluminum chloride. The results of the analysis for determination of total flavonoids correspond to the means \pm standard deviation of three repetitions. The samples of apples and onions presented higher content of flavonoid. The cooking process has affected the extraction of flavonoids in fruit by decreasing their levels, a fact explained by the presence of fibers, while it facilitated flavonoid extraction in the vegetables. Daily recommendation of flavonoids is not defined. However, as nutritionists know how food should be eaten and what their flavonoid levels are, they can prescribe and devise strategies and secure goals of intake, in order to promote the patient's health and hence also improve their social and economic condition.

Keywords: Flavonoids. Fruits. Vegetables. Organic Food. Crop Production. Food Handling.

\section{Introdução}

Flavonoides são compostos fenólicos que diferem entre si pela sua estrutura química, apresentam 15 átomos de carbono na forma C6 - C3 - C6, baseada no núcleo de dois anéis benzênicos, A e B, ligados a um anel pirano, C (Figura 1). ${ }^{1,2}$ Estão nos alimentos geralmente na forma de $O$-glicosídeos, com a molécula de açúcar ligada na posição 3 e, em alguns casos, na posição 7. A glicose, galactose, ramnose e frutose são os açúcares mais encontrados. ${ }^{1}$ 


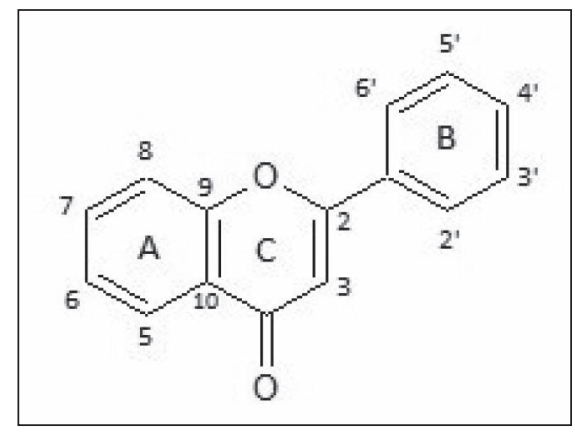

Figura 1. Estrutura básica dos flavonóides.

Segundo Pereira \& Cardoso, ${ }^{3}$ os flavonoides são metabólitos secundários. Foram identificadas mais de 8.000 substâncias pertencentes a este grupo. ${ }^{2}$ Esta variedade de compostos ocorre devido a uma grande combinação de diferentes açúcares e hidroxil $(\mathrm{OH})$ como substituintes na estrutura química básica. ${ }^{4}$ Os flavonoides utilizados na dieta humana são subdivididos em seis classes: Flavanonas, Flavonóis, Flavonas, Flavanóis, Isoflavonas e Antocianidinas., ${ }^{5,6}$

Os flavonoides estão relacionados com uma grande variedade de atividades biológicas, destacando-se a ação antioxidante, anti-inflamatória, antitumoral, antialérgica, antiviral, entre outras. ${ }^{1,78}$ A ação antioxidante dos flavonoides se dá pela sua capacidade de sequestrar radicais livres e quelar íons metálicos. ${ }^{4}$ Os flavonoides doam átomos de hidrogênio, protegendo assim os tecidos das reações provocadas por radicais livres e da peroxidação lipídica. ${ }^{9}$

A quercetina é o flavonoide mais abundante da dieta. Apresenta significativa ação antiinflamatória e potencial antioxidante. ${ }^{10} \mathrm{~A}$ ingestão média de flavonoides varia entre $26 \mathrm{mg}$ a $1 \mathrm{~g} /$ dia provenientes do consumo de fontes alimentares como frutas, verduras, chás, vinhos, grãos e sementes. ${ }^{11}$ Segundo Kozlowska \& Szostak-Wegierek, ${ }^{12}$ o consumo médio de flavonoides na população grega e espanhola varia entre $93 \mathrm{mg}$ e 126,1 mg, respectivamente. No Japão, o consumo médio pode chegar a $2 \mathrm{~g}$ devido ao alto consumo de legumes, soja e chá. Koehnlein, et al. ${ }^{13}$ verificaram que o consumo de flavonoides provenientes de preparações e alimentos de origem vegetal da dieta brasileira foi estimado em $374 \mathrm{mg} /$ dia. Esse estudo foi realizado com 37 alimentos de origem vegetal a partir do consumo alimentar da população brasileira e publicado na Pesquisa de Orçamento Familiar (POF) 2008/2009.14

De acordo com a POF (2008/2009) ${ }^{14}$ as frutas e hortaliças mais consumidas pela população da região Sul do Brasil são a maçã, melancia, banana d'água, laranja, pera, mamão, cebola, alface, tomate, repolho, mandioca e batata inglesa. Sendo assim, o objetivo do presente estudo foi avaliar a quantidade de flavonoides totais expressa em quercetina de algumas frutas e hortaliças mais 
consumidas pela população da região Sul do Brasil. Foram avaliados os teores de flavonoides em produtos convencionais e orgânicos, in natura, submetidos ao processo de cocção por calor seco em panela e micro-ondas.

\section{Material e Métodos}

\section{Material}

O estudo foi realizado no laboratório de Farmacognosia e Química II no Centro Universitário Autônomo do Brasil (UniBrasil) na Cidade de Curitiba - PR. Foram selecionadas duas frutas e duas hortaliças mais consumidas nos anos 2008/2009, segundo dados da POF. ${ }^{10}$ São elas: maçã, banana d'água, cebola e tomate, que podem ser consumidas in natura, pós-processo de cocção e microondas. Para análise, foram utilizadas três unidades de cada alimento, convencionais e orgânicos.

\section{Método}

A extração de flavonoides totais foi realizada de acordo com Alves \& Kubota, ${ }^{15}$ com modificações. Foram empregadas amostras in natura e amostras submetidas a processo de cocção em calor seco em panela e micro-ondas.

Para o preparo do extrato aquoso das amostras de cebola e tomate, primeiramente ambas foram lavadas em água corrente. As cascas da cebola foram retiradas e as amostras picadas com faca. Pesou-se $50 \mathrm{~g}$ de cada amostra picada. A de cebola in natura foi triturada em liquidificador, marca Walita, com $100 \mathrm{~mL}$ de água purificada por 5 minutos. A do tomate in natura foi triturada em liquidificador com $200 \mathrm{~mL}$ de água purificada por 5 minutos. Para a obtenção do extrato aquoso, o material resultante dos processos foi filtrado em gaze e o liquido obtido foi completado com água purificada para $200 \mathrm{~mL}$ e homogeneizado. Uma alíquota do extrato aquoso foi empregada para a determinação de flavonoides.

O processo de cocção da cebola consistiu em refogar sem óleo $50 \mathrm{~g}$ de cebolas picadas, em fogo baixo por 4 minutos e 30 segundos. Outras $50 \mathrm{~g}$ de amostra foram submetidas ao micro-ondas por 1 minuto na potência 100 watts. Para o tomate, o processo de cocção consistiu em refogar sem óleo $50 \mathrm{~g}$ de tomate picado, em fogo baixo por 2 minutos e 24 segundos. Outras $50 \mathrm{~g}$ de amostra foram submetidas ao micro-ondas por 40 segundos na potência 100 watts. Após, as amostras de cebola e tomate submetidas ao processo de cocção e micro-ondas também foram processadas da mesma forma que as in natura para a obtenção do extrato aquoso empregado para a avaliação de flavonoides. 
Para o preparo do extrato aquoso das amostras de banana e maçã, primeiramente as cascas das bananas foram retiradas, as maçãs lavadas em água corrente, as sementes foram extraídas e ambas as amostras foram picadas com faca. Pesou-se $50 \mathrm{~g}$ de cada amostra picada. A da banana in natura foi triturada em liquidificador, marca Walita, com $200 \mathrm{~mL}$ de água purificada por 5 minutos. A de maçã in natura foi triturada em liquidificador com $100 \mathrm{~mL}$ de água purificada por 5 minutos. Para a obtenção do extrato aquoso, o material resultante dos processos foi filtrado em gaze e o liquido obtido foi completado com água purificada para $200 \mathrm{~mL}$ e homogeneizado. Uma alíquota do extrato aquoso foi empregada para a determinação de flavonoides.

O processo de cocção da banana consistiu em refogar sem óleo $50 \mathrm{~g}$ de banana picada, em fogo baixo por 2 minutos. Outras $50 \mathrm{~g}$ de amostra foram submetidas ao micro-ondas por 30 segundos na potência 100 watts. Para a maçã, o processo de cocção consistiu em refogar $50 \mathrm{~g}$ de maçã picadas sem óleo, em fogo baixo por 2 minutos e 36 segundos. Outras $50 \mathrm{~g}$ de amostra foram submetidas ao micro-ondas por 50 segundos na potência 100 watts. Em seguida, as amostras da banana e maçã submetidas ao processo de cocção e micro-ondas também foram trituradas em liquidificador com a mesma quantidade de água e filtradas, e o líquido obtido, utilizado para a avaliação de flavonoides.

Foram misturados $2 \mathrm{~mL}$ de cloreto de alumínio a $2 \%(\mathrm{~m} / \mathrm{v})$ em $2 \mathrm{~mL}$ dos extratos aquosos. A absorbância foi determinada em $425 \mathrm{~nm}$ após dez minutos contra um branco, consistindo de uma solução de $2 \mathrm{~mL}$ de água purificada com $2 \mathrm{~mL}$ de cloreto de alumínio $2 \%$ (m/v). O conteúdo total de flavonoides foi determinado usando uma curva padrão de quercetina com concentrações entre 5 a $50 \mu \mathrm{g} / \mathrm{mL}$. Todos os ensaios foram realizados em triplicata e expressos por média \pm desvio padrão. ${ }^{15}$

\section{Análise Estatística}

Os resultados das análises de determinação de flavonoides totais correspondem à média \pm desvio padrão de três repetições, e foram comparadas por análise de variância (ANOVA) seguida do teste de Tukey para identificar as diferenças significativas entre as médias, utilizando o programa Sisvar, em que as médias no nível de $5 \%(\mathrm{p}<0,05)$ foram consideradas expressivas.

\section{Resultados e Discussão}

A curva de calibração para determinação de flavonoides obteve a equação da reta expressa por $y=0,0333 x-0,0096$, em que y é a absorbância e $\mathrm{x}$, a concentração de quercetina em $\mu \mathrm{g} / \mathrm{mL}$, com $\mathrm{R}^{2}=0,9997$. O cloreto de alumínio é um reagente empregado em espectrometria no UV-visível 
para a determinação estrutural de flavonoides. ${ }^{16}$ Atualmente, é utilizado para a determinação de flavonoides totais em própolis e plantas, usando como padrão a rutina ou quercetina. ${ }^{15,17} \mathrm{Na}$ reação, o íon alumínio $\mathrm{Al}^{3+}$ se complexa com as moléculas de flavonoides da amostra, estabelecendo o complexo estável flavonoide- $\mathrm{Al}^{3+}$ de coloração amarela cuja intensidade é proporcional à concentração de flavonoides presentes na amostra. ${ }^{16}$ Esta metodologia permite o doseamento de diferentes flavonoides. Portanto, no procedimento realizado no presente trabalho, utilizouse o flavonoide quercetina como padrão e assim os resultados obtidos foram flavonoides totais expressos em quercetina. Dessa forma, outros flavonoides, além da quercetina, estão presentes na absorção obtida.

Na tabela 1 encontram-se os resultados médios dos teores de flavonoides presentes nas hortaliças (cebola e tomate) e nas frutas (banana e maçã), cultivados de forma convencional e orgânica.

Tabela 1. Conteúdo de flavonoides presentes em hortaliças e frutas, convencionais e orgânicos, in natura, adquiridos na cidade de Curitiba - PR, no ano de 2015.

\section{Teores de Flavonoides}

Hortaliça/Fruta

Média \pm desvio padrão

$(\mathrm{mg} / \mathrm{l00g})$

Cebola convencional

Cebola orgânica

Tomate convencional

Tomate orgânico

Banana convencional

Banana orgânica

Maçã convencional

Maçã orgânica
$10,0 \pm 0,2$ a 3

$11,8 \pm 0,3$ a 4

$3,3 \pm 0,05$ a 1

$2,8 \pm 0,05$ a 1

$8,7 \pm 0,1$ a 2

$8,8 \pm 0,1$ a 2

$12,1 \pm 0,1$ a 4

$14,3 \pm 0,3$ a 5

A letra a seguida de número igual significa resultados estatisticamente iguais entre si.

A cebola orgânica apresentou maiores teores de flavonoides em relação à cebola convencional. Fato que pode ser justificado pelo não emprego de agrotóxicos no cultivo. Dessa maneira, produz concentrações superiores de compostos fenólicos, que atuam como agentes de defesa contra insetos, microrganismos, bactérias e fungos. ${ }^{18}$ 
Segundo o estudo de Arbos et al., ${ }^{19}$ os níveis de compostos fenólicos encontrados em hortaliças de cultivo orgânico foram maiores que os encontrados em hortaliças de cultivo convencional. Muitos fatores devem ser considerados em relação à qualidade nutricional dos alimentos orgânicos, como o tipo de produção, o tipo de sistema orgânico utilizado, fatores externos, como luz solar, chuva e temperatura, armazenamento e transporte. ${ }^{20}$ Já o tomate não apresentou diferença de teor de flavonoides entre o convencional e o orgânico. Indicando que no tomate os flavonoides presentes não influenciam tanto a defesa do vegetal quanto a cebola.

Nas frutas analisadas, a maçã apresentou maiores teores de flavonoides em relação à banana, e a amostra orgânica, maiores teores de compostos em relação à amostra convencional. A banana não apresentou diferença entre a amostra orgânica e a convencional, indicando, como no caso do tomate, que na banana os flavonoides presentes pouco influenciam a defesa do vegetal.

A biodiversidade das espécies resulta em variações de componentes básicos, como carboidratos, proteínas, lipídeos, fibra alimentar, minerais, vitaminas e também dos compostos bioativos, ${ }^{21}$ como flavonoides. Estes são produzidos em resposta a condições ambientais, por isso, pode-se explicar a diferença nos teores de flavonoides de uma mesma verdura em diferentes regiões. Vários estudos demonstraram que existem muitas variações no teor de flavonoides, ${ }^{22}$ uma vez que sua concentração pode aumentar em resposta ao estresse do meio ambiente, que pode ser causado por doenças, altitude, poluição atmosférica, nutrientes, clima e radiação ultravioleta (UV). ${ }^{23}$

Na tabela 2 estão descritos os resultados da comparação dos teores de flavonoides das hortaliças in natura e após os processos de cocção e micro-ondas.

Tabela 2. Conteúdo de flavonoides de hortaliças convencionais e orgânicas obtidas em Curitiba - PR, in natura, após processos de cocção, no ano de 2015.

\begin{tabular}{|c|c|c|c|c|}
\hline \multirow{3}{*}{ Processo } & $\begin{array}{c}\text { Cebola } \\
\text { convencional }\end{array}$ & $\begin{array}{c}\text { Cebola } \\
\text { Orgânica }\end{array}$ & $\begin{array}{c}\text { Tomate } \\
\text { convencional }\end{array}$ & $\begin{array}{c}\text { Tomate } \\
\text { Orgânico }\end{array}$ \\
\hline & & & & \\
\hline & $\begin{array}{c}\text { média } \pm \text { DP } \\
(\mathrm{mg} / 100 \mathrm{~g})\end{array}$ & $\begin{array}{c}\text { média } \pm \text { DP } \\
(\mathrm{mg} / 100 \mathrm{~g})\end{array}$ & $\begin{array}{c}\text { média } \pm \text { DP } \\
(\mathrm{mg} / 100 \mathrm{~g})\end{array}$ & $\begin{array}{c}\text { média } \pm \text { DP } \\
(\mathrm{mg} / 100 \mathrm{~g})\end{array}$ \\
\hline In natura & $10,06 \pm 0,2 \mathrm{a} 1$ & $11,80 \pm 0,3 \mathrm{a} 1$ & $3,36 \pm 0,05$ al & $2,86 \pm 0,05$ al \\
\hline Cocção & $10,83 \pm 0,05 \mathrm{a} 2$ & $12,16 \pm 0,2 \mathrm{a} 1$ & $5,06 \pm 0,05$ a 2 & $4,33 \pm 0,1 \mathrm{a} 2$ \\
\hline Micro-ondas & $11,0 \pm 0,4 \mathrm{a} 2$ & $12,40 \pm 0,6 \mathrm{a} 1$ & $5,73 \pm 0,1 \mathrm{a} 3$ & $4,50 \pm 0,1 \mathrm{a} 2$ \\
\hline
\end{tabular}

A letra a seguida de número igual na mesma coluna significa resultados estatisticamente iguais entre si. 
A cebola convencional in natura apresentou valor inferior ao processo de cocção por calor seco em panela e micro-ondas, em que o aquecimento permitiu uma melhor extração dos flavonoides. Em estudo de Ioku et al., ${ }^{24}$ que avaliaram métodos de cocção para dosar os flavonoides em cebola, observou-se que, após submeter a cebola sob o calor de micro-ondas, durante 1 minuto houve aumento em 1,5 vez no teor total de quercetina, que é o principal flavonoide presente na dieta humana e encontrado na cebola, mostrando então que os compostos foram extraídos com mais facilidade, porém, quando foi submetido à cocção em água, houve perda significativa dos compostos. Tal fato é justificado porque os flavonoides migram para a água empregada na cocção. Verifica-se que cebolas cozidas ou assadas apresentam uma melhor extração dos flavonóis. ${ }^{25}$

No presente estudo, houve um aumento no teor de flavonoides, pois para a análise foi utilizado calor seco, facilitando a extração, não deixando os mesmos serem degradados ou lixiviados na água de cocção. ${ }^{26}$ A cebola orgânica in natura e submetida ao processo de cocção por calor seco em panela e micro-ondas apresentou o mesmo resultado, o que significa que o processo não diminui os teores de flavonoides.

O tomate convencional e o orgânico in natura apresentaram menores valores de flavonoides em relação ao processo de cocção a seco em panela e micro-ondas, pois nesse caso, o processo de aquecimento também permitiu uma melhor extração dos flavonoides nas amostras.

Na tabela 3, observa-se o resultado da comparação dos teores de flavonoides das frutas in natura e após os processos de cocção e micro-ondas. Para a banana convencional, as amostras in natura e submetidas ao processo de cocção em panela apresentaram maiores teores de flavonoides em relação à amostra submetida ao processo de cocção em micro-ondas. A banana orgânica apresentou maior teor de compostos nas amostras in natura e submetidas ao processo de cocção em micro-ondas em relação à amostra submetida ao processo de cocção em panela. Para as maçãs, observou-se que tanto a convencional como a orgânica tiveram valores superiores de flavonoides na amostra in natura em relação às amostras submetidas em cocção na panela e micro-ondas. 
Tabela 3. Conteúdo de flavonoides de frutas convencionais e orgânicas obtidas em Curitiba - PR, in natura, após processos de cocção, no ano de 2015.

\begin{tabular}{ccccc}
\hline Processo & $\begin{array}{c}\text { Banana } \\
\text { convencional }\end{array}$ & $\begin{array}{c}\text { Banana } \\
\text { Orgânica } \\
\text { média } \pm \mathrm{DP} \\
(\mathrm{mg} / 100 \mathrm{~g})\end{array}$ & $\begin{array}{c}\text { Maçã } \\
\text { Convencional } \\
(\mathrm{mg} / 100 \mathrm{~g})\end{array}$ & $\begin{array}{c}\text { Maçã } \\
\text { Orgânica }\end{array}$ \\
\hline In natura & $8,70 \pm 0,1 \mathrm{a} 2$ & $8,8 \pm 0,1 \mathrm{a} 2$ & $12,13 \pm 0,1 \mathrm{a} 3$ & $14,30 \pm 0,3 \mathrm{a} 3$ \\
Cocção & $8,80 \pm 0,1 \mathrm{a} 2$ & $6,63 \pm 0,4 \mathrm{al}$ & $8,93 \pm 0,05 \mathrm{a} 1$ & $9,96 \pm 0,3 \mathrm{a} 1$ \\
Micro-ondas & $7,86 \pm 0,3 \mathrm{a} 1$ & $8,06 \pm 0,1 \mathrm{a} 2$ & $11,43 \pm 0,1 \mathrm{a} 2$ & $10,93 \pm 0,2 \mathrm{a} 2$ \\
\hline
\end{tabular}

A letra a seguida de número igual na mesma coluna significa resultados estatisticamente iguais entre si.

No presente estudo, notou-se que a extração de flavonoides das frutas e hortaliças foi afetada pelos processos de cocção empregados. No caso das frutas, o calor seco influenciou negativamente, dificultando a extração de flavonoides, enquanto nas hortaliças, positivamente, facilitando a extração. Possivelmente, a presença das fibras nas frutas dificultou a extração dos flavonoides quando as mesmas foram aquecidas. Este aquecimento promoveu um aprisionamento dos flavonoides na fibra. As fibras são carboidratos complexos, podendo ser solúveis e insolúveis. As solúveis reduzem o tempo de trânsito gastrointestinal e a absorção enteral do colesterol. São representadas pela pectina nas frutas, ou pelas gomas na aveia, cevada e leguminosas. ${ }^{27} \mathrm{Um}$ dos mecanismos que podem explicar a ação das fibras solúveis, como a pectina, seria por estas absorverem água e formarem um gel no lúmen intestinal, atuando na redução de absorção dos carboidratos e diminuindo a reabsorção dos ácidos biliares. ${ }^{28}$ Dessa forma, tal como ocorre com os carboidratos e outros nutrientes, a fibra torna os flavonoides menos disponíveis para a absorção.

A pectina é uma fibra com alto poder geleificante após aquecimento, e é encontrada em maior número em maçãs e bananas. Alguns estudos mostram que o acúmulo de pectina em maçãs e bananas frescas apresenta uma concentração maior 0,5-1,6\% e 0,7-1,2\% da fibra, respectivamente. O tomate apresenta uma concentração menor $0,2-0,6 \%$ da fibra. ${ }^{29}$ A presença de concentrações maiores de fibras na banana e na maçã justifica o fato de que pelo menos em algum dos processos de cocção houve uma menor extração de flavonoides, pois os mesmos podem ter ficado retidos na fibra geleificada pelo aquecimento.

Nesta pesquisa, o aquecimento das hortaliças permitiu uma melhor extração dos flavonoides, pois a cebola e o tomate não são alimentos com alto teor de fibras e estas parecem não interferir na extração. Nesse caso, a cocção amolece esses alimentos, favorecendo a extração dos flavonoides. 
Sendo assim, a coç̧ão de frutas como banana e maçã dificulta a absorção dos flavonoides no trato intestinal, indicando que a forma ideal de consumo dessas frutas é in natura. Já para as hortaliças, foi verificado que o aquecimento favorece a extração de flavonoides. Portanto, o consumo de tomate e cebola pode ser tanto in natura como sob cocção.

Segundo estudo de Arabbi et al., ${ }^{30}$ em relação à população brasileira, a ingestão estimada de flavonoides variou de 60 a $106 \mathrm{mg} / \mathrm{dia}$, com média de ingestão entre as mulheres de $79 \mathrm{mg} /$ dia e entre os homens, de $86 \mathrm{mg} / \mathrm{dia}$. Essa ingestão de flavonoides foi estimada de acordo com o consumo de alimentos, baseado na composição dietética obtida de várias pesquisas dietéticas e de hábitos alimentares realizadas e disponíveis no país. Dessa forma, a ingestão de flavonoides é bem variada, podendo ser explicada pelos diferentes hábitos alimentares. Em outro estudo realizado por Corrêa et al., ${ }^{31}$ verificou-se que a população brasileira consome em média 138,92 mg/dia de flavonoides. Foi constatado que a ingestão de compostos fenólicos como os flavonoides é baixa, em virtude do consumo insuficiente de frutas e hortaliças. Ressalta-se que o café e o feijão preto são os principais alimentos que contribuem para o consumo de compostos fenólicos no país.

A ingestão diária de flavonoides é ainda pouco documentada, devido à falta de dados do conteúdo de flavonoides nos alimentos. O comitê do Food and Nutrition Board, da US National Academy of Sciences, não esclarece as DRIs desses compostos. ${ }^{32} \mathrm{O}$ conhecimento de como as hortaliças e frutas devem ser consumidas e dos teores de flavonoides presentes possibilita ao nutricionista prescrever e traçar estratégias e metas seguras de ingestão, para promoção da saúde do paciente.

\section{Conclusão}

Os resultados do presente estudo demonstram que houve diferença quanto ao processo de cultivo na cebola e na maçã. Foi observado que essas amostras apresentaram maior conteúdo de flavonoides. Fato justificado pelo mecanismo dessas plantas de produzir flavonoides para sua proteção contra os estresses do ambiente. Já a banana e o tomate não apresentaram diferença entre o cultivo orgânico e o convencional, indicando que os flavonoides presentes possivelmente não influenciam tanto a defesa do vegetal quanto a cebola e a maçã.

Com os achados da pesquisa, foi possível verificar que houve diferença na extração de flavonoides totais dos alimentos in natura e submetidos ao processo de cocção. No caso das hortaliças, o calor seco influenciou, facilitando a extração de flavonoides, enquanto nas frutas, dificultou a extração. Possivelmente, a presença da fibra pectina nas frutas atuou diminuindo a extração dos flavonoides, quando as mesmas foram aquecidas. Sendo assim, as frutas devem ser consumidas in natura, pois o calor seco dificulta a extração dos flavonoides e a absorção dos mesmos no trato digestório. 
Por meio do conhecimento de como os alimentos devem ser consumidos e dos teores de flavonoides, o profissional nutricionista pode prescrever e traçar estratégias e metas seguras de ingestão, para promoção da saúde do paciente, visando também a sua condição social e econômica. Destacando a importância do consumo de alimentos entre os grupos de frutas e hortaliças, com o intuito de aumentar a contribuição de flavonoides na dieta brasileira. Existem poucos dados da literatura sobre o assunto, o que implica a necessidade de serem analisados mais alimentos e de se obter mais valores sobre esses compostos, para que futuramente se estabeleça uma recomendação, para uma prescrição mais segura.

\section{Colaboradores}

Savi PRS participou da concepção, da análise, interpretação dos dados e versão final do artigo; Santos L, da concepção, da análise, interpretação dos dados e versão final do artigo; Biesek S, do desenho do estudo da redação do artigo e da revisão e versão final; Macedo A, da análise do estudo; Lima CP trabalhou em todas as etapas, desde o desenho e concepção do estudo até a revisão da versão final do artigo.

Conflito de Interesses: Os autores declaram não haver conflito de interesses.

\section{Referências}

1. Bernardes NR, Pessanha FF, Oliveira DB. Alimentos funcionais: Uma breve revisão. Ciência e Cultura - Rev. Cient. Multid. Cent. Univ. da FEB 2010; 6(2): 11-19.

2. Koirala N, Thuan NH, Ghimire GP, Thang DV, Sohng KJ. Methylation of flavonoids: Chemical structures, bioactivities, progress and perspectives for biotechnological production. Enzyme Microb Tech. 2016; 86: 103-116.

3. Pereira RJ, Cardoso MG. Metabólitos secundários vegetais e benefícios antioxidantes. J. of Biot. and Biod. 2012; 3(4): 146-152.

4. Vinayagam R, Xu B. Antidiabetic properties of dietary flavonoids: a cellular mechanism review. Nutr Metab. 2015; 12(60): 1-20.

5. Johnston GAR. Flavonoid nutraceuticals and ionotropic receptors for the inhibitory neurotransmitter GABA. Neurochem Int. 2015; 89: 120-125.

6. Hoensch HP, Oertel R. The value of flavonoids for the human nutrition: Short review and perspectives. Clin Nutr Exp. 2015; 3: 8-14.

7. Pereira RJ, Cardoso MG. Metabólitos secundários vegetais e benefícios antioxidantes. J. of Biot. and Biod. 2012; 3(4): 146-152. 
8. Cho JG, Song NY, Nam TG, Shrestha S, Park HJ, Lyu HN, et al. Flavonoids from the Grains of C1/R-S Transgenic Rice, the Transgenic Oryza sativa spp. japonica, and Their Radical Scavenging Activities. J. Agric. Food Chem. 2013; 61(43): 10354-10359.

9. Kumar S, Pandey AK. Chemistry and Biological Activities of Flavonoids: An Overview. Sci World J Journal. 2013; 2013: 1-16.

10. Nabavi SF, Russo GL, Daglia M, Nabavi SM. Role of quercetin as an alternative for obesity treatment: You are what you eat! Food Chem. 2015; 179(15): 305-310.

11. Schwertz MC, Maia JRP, Sousa RSF, Aguiar JPL, Yuyama LKO, Lima ES Efeito hipolipidêmico do suco de camu-camu em ratos. Rev. de Nutr. 2012; 25(1): 35-44.

12. Kozlowska A, Szostak-Wegierek, D. Flavonoids-Food sources and health benefits. Roc. Państ. Zakł. Hig. J. 2014; 65(2): 79-85.

13. Koehnlein EA, Koehnlein EM, Peralta RM. Estimativa do consumo de fenólicos e da capacidade antioxidante dos principais alimentos e preparações de origem vegetal da dieta brasileira. Nutrire Rev. da Soc. Bras. de Nutr. 2013; 38: 317-317.

14. Instituto Brasileiro de Geografia e Estatística. Pesquisa de Orçamentos Familiares 2008-2009: Aquisição Familiar Domiciliar Per Capita, Rio de Janeiro, 2010.

15. Alves E, Kubota EH. Conteúdo de fenólicos, flavonoides totais e atividade antioxidante de amostras de própolis comerciais. Rev Ciênc Farm Básica Apl. 2013; 34(1): 37-41.

16. Silva LAL, Pezzini BR, Soares L. Spectrophotometric determination of the total flavonoid content in Ocimum basilicum L. (Lamiaceae) leaves. Pharmacogn Mag. 2015; 11(41): 96-101.

17. Peixoto Sobrinho TJS, Gomes, TLB, Cardoso KCM; Albuquerque UP, Amorim, ELC. Teor de flavonoides totais em produtos contendo pata-de-vaca (Bauhinia L.) comercializados em farmácias de Recife/PE. Rev. Bras. de Pl. Med. 2012; 14(4): 586-591.

18. Silva MLC, Renata Silva Costa, Santos Santana AS, Koblitz MGB. Phenolic compounds, carotenoids and antioxidant activity in plant products. Semin: Cien. Agrar. 2010; 31(3): 669-682.

19. Arbos KA, Freitas RJS, Stertz SC; Dornas MF. Atividade antioxidante e teor de fenólicos totais em hortaliças orgânicas e convencionais. Ci. e Tec. de Alim. 2010; 30(2): 501-506.

20. Souza AA, Azevedo E; Lima E; Silva APF. Alimentos Orgânicos e Saúde Humana: Estudo sobre as controvérsias. Rev. Panam. de Sal. Púb. 2012; 31(6): 513-517.

21. Toledo A, Burlingame B. Biodiversity and nutrition: a common path toward global food security and sustainable development. J. of Fo. Comp. and Anal. 2006; 19: 477-483.

22. Gobbo-Neto L, Lopes NP. Plantas Medicinais: Fatores de Influência no conteúdo de metabólitos secundários. Quim. Nova. 2007; 30(2): 374-381.

23. Macedo JM, Souza LGP, Valenzuela VCT, Oliveira AB, Castilho RO; Jácome RLRP. Variação sazonal nos teores de flavonoides, taninos e atividade antioxidante de Davilla rugosa Poir. Rev Ciênc Farm Básica Apl. 2013; 34(4): 585-590.

24. Ioku K, Aoyama Y, Tokuno A, Terao J, Nakatani N, Takei Y. Various Cooking Methods and the Flavonoid Content in Onion. J. of Nurt. al Sci. and Vitam. 2001; 47: 78-83. 
25. Fabbrin ADT, Crosby GA. A review of the impact of preparation and cooking on the nutritional quality of vegetables and legumes. Int J Gastr Food Sci. 2016; 3: 2-11.

26. Melo, EA, Maciel MLS, Lima VLAG, Santana APM. Capacidade antioxidante de hortaliças submetidas a tratamento térmico. Rev. da Soc. Bras. de Alim. e Nutr. 2009; 34(1): 85-95.

27. Sociedade Brasileira de Cardiologia. IV Diretriz Brasileira sobre Dislipidemias e Prevenção da Aterosclerose. Arq. Bras. de Card. 2007; 88 (1 Supl 1):1-19.

28. Ma MM, Um TH. Effects of extraction methods and particle size distribution on the structural, physicochemical, and functional properties of dietary fiber from deoiled cumin. Food Chem. 2016; 194: 237-246.

29. Canteri MHG, Moreno L, Wosiacki G, Scheer, AP. Pectina: da matéria prima ao produto final. Polímeros. 2012; 22(2): 149-157.

30. Arabbi PR, Genovese MI, Lajolo FM. Flavonoids in vegetable foods commonly consumed in Brazil and estimated ingestion by the Brazilian population. J. of Agr. and Fo. Chem. 2004; 52(5):1124-1131.

31. Corrêa VG, Tureck C, Gelvani L, Peralta MR, Koehnlein EA. Estimate of consumption of phenolic compounds by Brazilian population. Rev. Nutr. 2015; 28(2): 185-196.

32. Williamson G, Holst B. Dietary reference intake (DRI) value for dietary polyphenols: are we heading in the right direction?. Bris. J. of Nutr. 2008; 99(3): S55-58. 
